\title{
Hábitos alimentarios de individuos inmaduros de Ephemeroptera, Plecoptera y Trichoptera en la parte media de un río tropical de montaña
}

\author{
Cristian José Guzmán-Soto \& Cesar Enrique Tamarís-Turizo \\ Grupo de Investigación en Limnología Neotropical, Universidad del Magdalena, Instituto de Investigaciones \\ Tropicales-INTROPIC, Colombia; guzmansotocj@gmail.com; cesartamaris@yahoo.es
}

\author{
Recibido 12-XII-2013. Corregido 20-I-2014. Aceptado 13-II-2014.
}

\begin{abstract}
Feeding habits of immature individuals of Ephemeroptera, Plecoptera, and Trichoptera from middle reaches of a tropical mountain stream. Morphological and behavioral aspects of insects allow their grouping in trophic guilds and represent their dependence on food resources. We determined the feeding habits of immature organisms of Ephemeroptera, Plecoptera, and Trichoptera (EPT) from the middle reaches of Gaira stream (Sierra Nevada de Santa Marta, Colombia), using gut content analysis. We identified 13 EPT genera, but only ten were analyzed for a total of 100 organisms. We describe six food items: animal parts (AP), vascular plant tissue (VPT), microalgae (M), fungi (F), coarse particulate organic matter (CPOM) and fine particulate organic matter (FPOM). Baetodes was determined to be a collector-scraper, since FPOM represented $46.6 \%$ of food content, followed by F (38.4\%). Chimarra, Leptohyphes, Lachlania, and Thraulodes were categorized as collectors with average proportions of FPOM 86.8\%, 93.1\%, 93.1\% and 93.7\%, respectively. Phylloicus, Smicridea and Leptonema were main consumers of VPT and CPOM with proportions of $76.3 \%, 54.6 \%$, and $62.4 \%$, respectively; while ratios of FPOM were $22.3 \%, 38.8 \%$, and $32.9 \%$, respectively. While all are detritivores, Phylloicus is functionally classified as shredders and Smicridea and Leptonema as collectors. Atopsyche and Anacroneuria were the only taxa in which AP were observed in high proportions, $57.9 \%$ and $58.2 \%$, respectively, for that reason they were classified as predators. The organisms examined consume a wide variability of resources. Rev. Biol. Trop. 62 (Suppl. 2): 169-178. Epub 2014 April 01.
\end{abstract}

Key words: aquatic insects, gut contents, food item, guild, Gaira stream.

El conocimiento de los hábitos alimentarios de los organismos en los ecosistemas lóticos contribuye a una mejor comprensión de las actividades naturales de tales ambientes (Cummins, 1973). En efecto, las funciones ecológicas de los macroinvertebrados se pueden describir a través de atributos asociados a las especies que reflejan sus adaptaciones a las condiciones ambientales (Townsend \& Hildrew, 1994). La asignación de especies a gremios tróficos está basada en el tipo de alimento ingerido (Cummins, 1973). Por otro lado, aspectos morfológicos y de comportamiento permiten agrupar a los insectos acuáticos en diferentes grupos funcionales de alimentación, y representar la dependencia de la comunidad lotica hacia recursos alimentarios particulares, permitiendo detectar patrones de cambio, desde la zona de cabecera hasta los tramos inferiores (Vannote, Minshall, Cummings, Sedell \& Cushing, 1980).

Existen dos clasificaciones principales usadas para describir la función de los insectos acuáticos en sus ecosistemas según su alimentación. Cummins (1973) describió los grupos funcionales alimentarios, los que se basan en el comportamiento de alimentación. Estos grupos incluyen: fragmentadores, recolectores, raspadores y depredadores. Por otro lado, los insectos pueden ser clasificados en gremios tróficos según el alimento consumido. Estos pueden ser: detritívoros, herbívoros, y depredadores. Aunque algunos trabajos tienden a combinar ambas clasificaciones, es importante distinguir 
entre ellas. Los grupos funcionales se enfocan en función (raspador: raspar el sustrato) y los gremios en el alimento consumido (detrito o tejido vivo) sin importar la forma de consumo.

Entre los insectos acuáticos, los ordenes Ephemeroptera, Plecoptera y Trichoptera han recibido mayor atención por parte de los investigadores, por ser abundantes y diversos (Wiggins \& Mackay, 1978; Merritt \& Cummins, 1996; Stewart \& Stark, 2002; MuñozQuesada, 2004). Los Ephemeroptera son un pequeño grupo de insectos en cuanto al número de géneros (375) con 3000 especies descritas en el ámbito mundial (Domínguez, Molineri, Pescador, Hubbard \& Nieto, 2006). Sin embargo, son un componente conspicuo del bentos en sus etapas inmaduras, lo que le confiere su importancia en las cadenas alimentarias, en las que llenan una diversidad de funciones, desde depredadores hasta raspadores (Merritt \& Cummins, 1996; Domínguez et al., 2006). Los Plecoptera también son un pequeño grupo, con cerca de 3200 especies descritas (Stark, Froehlich \& Zúñiga, 2009). Este grupo se ha caracterizado por desempeñar principalmente la función de depredadores (Tamarís-Turizo \& Sierra-Labastidas, 2009; Zúñiga, 2010). Los Trichoptera son importantes debido al alto número de especies (13 574) (Morse, 2011), y su abundancia y distribución biogeográfica (Wiggins, 1996). Tienen una marcada importancia en las cadenas tróficas, por la abundancia de algunas especies y la variedad de nichos que ocupan las larvas en diferentes grupos, como depredadores hasta recolectores (Wiggins \& Mackay, 1978; Muñoz-Quesada, 2004).

Debido al limitado conocimiento sobre el papel trófico que cumplen los macroinvertebrados en la transformación de la materia orgánica en los ecosistemas acuáticos tropicales, se evaluaron los hábitos alimentarios en tres órdenes (Ephemeroptera, Plecoptera y Trichoptera) que presentan altas abundancias en el río Gaira (Sierra Nevada de Santa Marta - SNSM). Además, con el fin de conocer si los gremios tróficos asignados en este estudio difieren a lo reportado para otras regiones, como lo sugiere Tomanova, Goitia \& Helesic (2006) y
Chará-Serna, Chará, Zúñiga, Pedraza \& Giraldo (2010); los resultados fueron comparados con otros trabajos en ríos de zonas templadas.

\section{MATERIALES Y MÉTODOS}

Área de estudio: El río Gaira tiene una longitud aproximada de $32.53 \mathrm{~km}$, se extien-

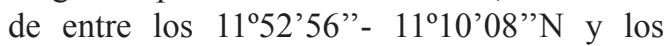
$74^{\circ} 46^{\prime} 22^{\prime}$ '- 7401'07' W, Colombia. El sector de Honduras, sitio de los muestreos se ubica en la parte media del río a $900 \mathrm{msnm}$. Se caracteriza por presentar una temperatura promedio anual de $15^{\circ} \mathrm{C}$. En el bosque ribereño dominan especies en el estrato arbóreo superior $(>24 \mathrm{~m})$, con entrelazamiento de las copas de los mismos, dando como resultado una cobertura cerrada del dosel. El cauce del río en esta zona se caracteriza por ser pedregoso, lo cual genera zonas de corrientes rápidas, además presenta zonas de lentos y pequeñas pozas, produciendo una heterogeneidad de microhábitats.

Se realizó un muestreo en los meses de abril, junio, agosto y diciembre de 2010. En cada periodo de muestreo se recolectaron cuatro muestras de hojarasca de $500 \mathrm{~g}$ utilizando una red de mano, con una abertura de malla de $200 \mu \mathrm{m}$, en un tramo de $100 \mathrm{~m}$ en el cual se examinaron zonas de rápidos y pozas (Darrigan, Vilches, Legarralde \& Damborenea, 2007). Las muestras fueron preservadas en bolsas plásticas con etanol al 96\% y transportadas al laboratorio para su posterior análisis.

Los insectos fueron identificados hasta nivel de género utilizando las claves taxonómicas y guías de: Merritt \& Cummins (1996), Wiggins (1996), Fernández \& Domínguez (2001), Posada-García \& Roldán-Pérez (2003), Wiggins (2004), Pes, Hamada \& Nessimian (2005), Domínguez et al. (2006), Domínguez \& Fernández (2009), Stark et al. (2009). Posteriormente, se procedió a medir la longitud total del cuerpo (LT) (Cuadro 1) que comprendió desde el ápice de la cabeza hasta la parte terminal del abdomen (Reynaga, 2009). El análisis del contenido de los tractos digestivos se realizó en organismos de tallas promedio, empleando la técnica propuesta por Tomanova, 
CUADRO 1

Composición porcentual (\%) de la dieta y tallas promedio de los diez géneros evaluados de Trichoptera, Plecoptera y Ephemeroptera

TABLE 1

Percentage composition (\%) of diet and average length of the ten genera evaluated Trichoptera, Plecoptera and Ephemeroptera

\begin{tabular}{|c|c|c|c|c|c|c|c|c|c|}
\hline Orden & Género & $\begin{array}{c}\text { No Ind } \\
\text { analizados }\end{array}$ & $\mathrm{LT} \pm \mathrm{DE}(\mathrm{mm})$ & RA & $\mathrm{TPV}$ & MA & $\mathrm{HN}$ & MOPG & MOPF \\
\hline \multirow[t]{5}{*}{ Trichoptera } & Atopsyche & 2 & $8.57 \pm 1.91$ & 58.56 & 1.97 & 0.48 & 0.75 & 18.82 & 19.42 \\
\hline & Chimarra & 5 & $4.53 \pm 1.97$ & 0 & 0 & 1.99 & 0 & 11.19 & 86.82 \\
\hline & Phylloicus & 8 & $13.73 \pm 1.79$ & 0.57 & 11.26 & 0.78 & 0.02 & 65.04 & 22.32 \\
\hline & Smicridea & 7 & $15.71 \pm 1.22$ & 1.07 & 24.05 & 2.91 & 0.67 & 38.39 & 32.91 \\
\hline & Leptonema & 8 & $16.38 \pm 1.59$ & 1.19 & 20.67 & 4.66 & 0.68 & 34.02 & 38.77 \\
\hline Plecoptera & Anacroneuria & 36 & $6.13 \pm 0.88$ & 57.2 & 1.86 & 0.17 & 0.67 & 21.58 & 18.51 \\
\hline \multirow[t]{4}{*}{ Ephemeroptera } & Baetodes & 5 & $3.5 \pm 0.53$ & 0.63 & 0.27 & 0.19 & 38.97 & 13.38 & 46.56 \\
\hline & Lachlania & 10 & $13.16 \pm 1.89$ & 0.02 & 2.66 & 0.78 & 0.01 & 2.8 & 93.73 \\
\hline & Thraulodes & 6 & $6.88 \pm 1.05$ & 0 & 2.09 & 1.51 & 0 & 3.27 & 93.13 \\
\hline & Leptohyphes & 13 & $4.08 \pm 0.98$ & 0.73 & 0.73 & 0.13 & 0.95 & 4.39 & 93.08 \\
\hline
\end{tabular}

Los ítems alimentarios se clasificaron en: Restos animales (RA), Tejido de planta vascular (TPV), Microalgas (MA), Hongos $(\mathrm{HN})$, Detritos gruesos (MOPG) y Detritos finos (MOPF). Para las tallas corporales se tuvo en cuenta la medición de la Longitud total (LT) expresada en mm con una desviación estándar (DE).

Goitia \& Helesic (2006). Se extrajo el tracto de uno a diez individuos de cada taxón (de acuerdo a su abundancia), los cuales fueron combinados y homogeneizados sobre un portaobjeto con glicerina. Se examinaron 20 campos de manera aleatoria con un microscopio (Nikon EC100) y posteriormente se determinó el área relativa promedio de cada ítem con la ayuda de una cámara fotográfica ajustada al microscopio para describir y cuantificar el área ocupada en el tracto digestivo.

Los ítems alimentarios del contenido estomacal fueron categorizados de acuerdo al tamaño de los materiales observados y los gremios tróficos fueron asignados según el ítem con mayor proporción promedio, según los criterios de Guiller \& Malmqvist (1998). Se analizó el contenido estomacal de 100 organismos distribuidos en diez géneros (Cuadro 1). Los géneros Helicopsyche (Helichopsychidae), Nectopsyche (Leptoceridae) y Tricorythodes (Leptohyphidae) no se evaluaron, ya que sus tractos digestivos se encontraron vacíos. Se identificaron en total seis ítems: Tejido de Restos Animales (RA), Tejido de Plantas
Vasculares (TPV), Microalgas (MA), Hongos (HN), Materia Orgánica Partículada Gruesa (MOPG) y Materia Orgánica Partículada Fina (MOPF) (Fig. 1).

Análisis estadístico: Se realizó un Análisis de Componentes Principales (ACP) para conocer las tendencias de los posibles hábitos alimentarios de los insectos evaluados a través de los ítems más frecuentes por taxón (Legendre \& Legendre, 1998). A partir de la matriz de datos porcentuales se construyó una matriz comparativa entre los géneros, con previa transformación de los datos a Arcoseno $\sqrt{(\mathrm{X} i / 100)}$ (Sokal \& Rohlf, 1995). Se usó el método Unweighted Pair Group Method with Arithmetic Mean (UPGMA) para sugerir una clasificación alimentaria (Sneath \& Sokal, 1973; Wantzen \& Rueda, 2009). Finalmente, para comprobar las posibles diferencias estadística de los contenidos estomacales dominantes entre géneros (Clarke \& Gorley, 2005) se realizó un Análisis multivariado de varianza no paramétrico-NPMANOVA, $\mathrm{p}<0.05$ (Anderson, 2001). Los análisis efectuados se realizaron 


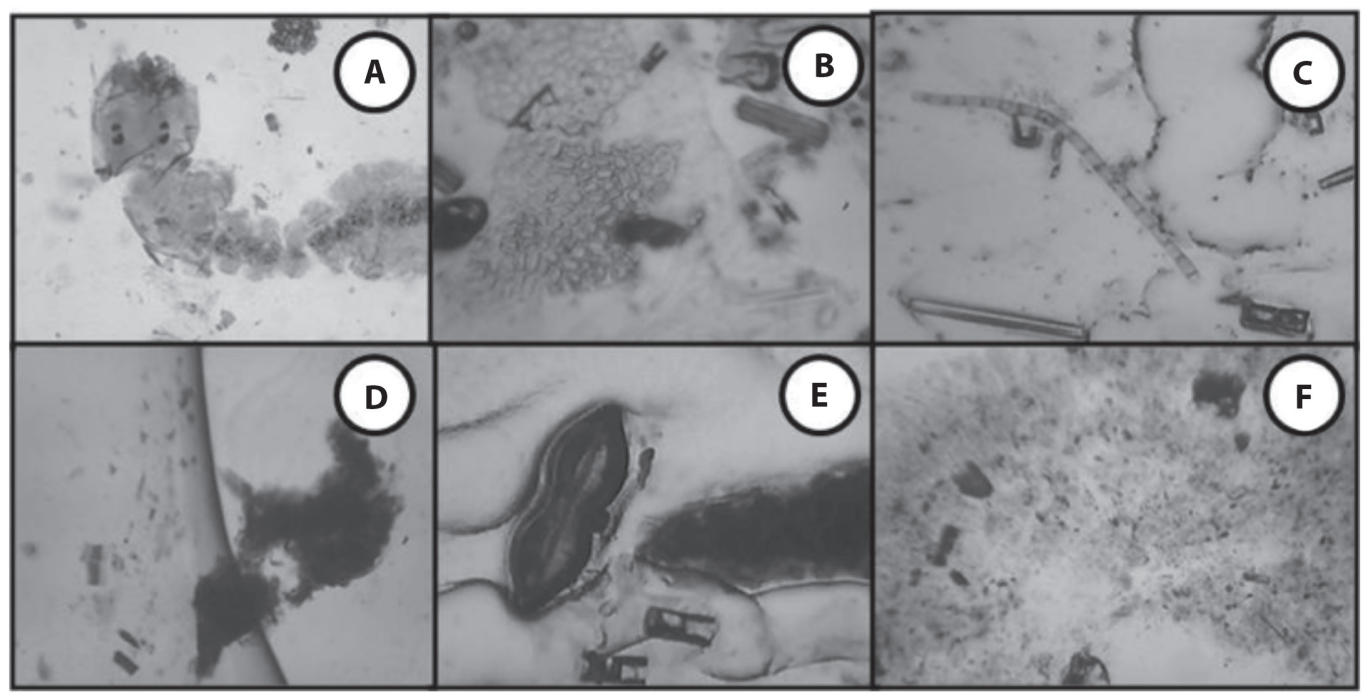

Fig. 1. (A). Restos animales (Simulium) encontrados en el intestino de Anacroneuria (40x). (B). Tejido de Plantas Vasculares encontrado en el intestino de Leptonema (10x). (C). Hongo (Hifa) encontrado en el intestino de Smicridea (40x). (D). Materia Orgánica Particulada Gruesa (MOPG) encontrada en el intestino de Leptonema (40x). (E). Microalga (Surirella) encontrada en el intestino de Smicridea (40x). (F) Materia Orgánica Particulada Fina (MOPF) encontrada en el intestino de Lachlania (40x).

Fig. 1. (A). Animal Parts (Simulium) found in gut of Anacroneuria (40x). (B). Vascular Plant tissue found in gut of Leptonema (10x). (C). Fungi (hyphae) found in gut of Smicridea (40x). (D). Coarse Particulate Organic Matter (CPOM) found in gut of Leptonema (40x). (E). Microalgae (Surirella) found in gut of Smicridea (40x). (F) Fine particulate organic matter (MOPF) found in gut of Lachlania (40x).

usando PRIMER® Versión DEMO (6.1.2) Plymouth Routines in Multivariate Ecological Research (Clarke \& Gorley, 2005) y PAST (Hammer, 2001).

\section{RESULTADOS}

Se recolectaron 68 individuos del orden Ephemeroptera, cuya abundancia correspondió al $10 \%$ aproximadamente de los organismos recolectados, donde Leptohyphes (Leptohyphidae) fue el género más abundante con 30 organismos seguido de Lachlania (Oligoneuriidae) con 17, Thraulodes (Leptophlebiidae) con 12, Baetodes (Baetidae) seis y por último tres del género Tricorythodes (Leptohyphidae). Mientras que el orden Plecoptera solo se encontró el género Anacroneuria (Perlidae). Este grupo registró las mayores abundancias, con 275 individuos, cerca del $50 \%$ de la abundancia de los tres grupos. Finalmente, el orden Trichoptera presentó cerca del $40 \%$ de la muestra de los órdenes estudiados, siendo Smicridea (Hydropsychidae) el género más abundante con 89 individuos seguido de Leptonema (Hydropsychidae) con 79, Phylloicus (Calamoceratidae) con 34 organismos, mientras que Chimarra (Philopotamidae), Nectopsyche (Leptoceridae) y Atopsyche (Hydrobiosidae) registraron las abundancias más bajas con siete, cuatro y dos individuos, respectivamente. Del género Helicopsyche (Helichopsychidae) se encontró solo un ejemplar durante el estudio (Fig. 2).

Clasificación trófica: El análisis de clasificación (UPGMA) de los géneros evaluados, permitió reconocer cuatro grupos claramente diferenciados de acuerdo al tipo de alimentación, lo cual coincide con los ítems alimentarios identificados y representados en el ACP (Fig. 3). Los géneros se ubicaron en los siguientes grupos: Grupo A: conformado 


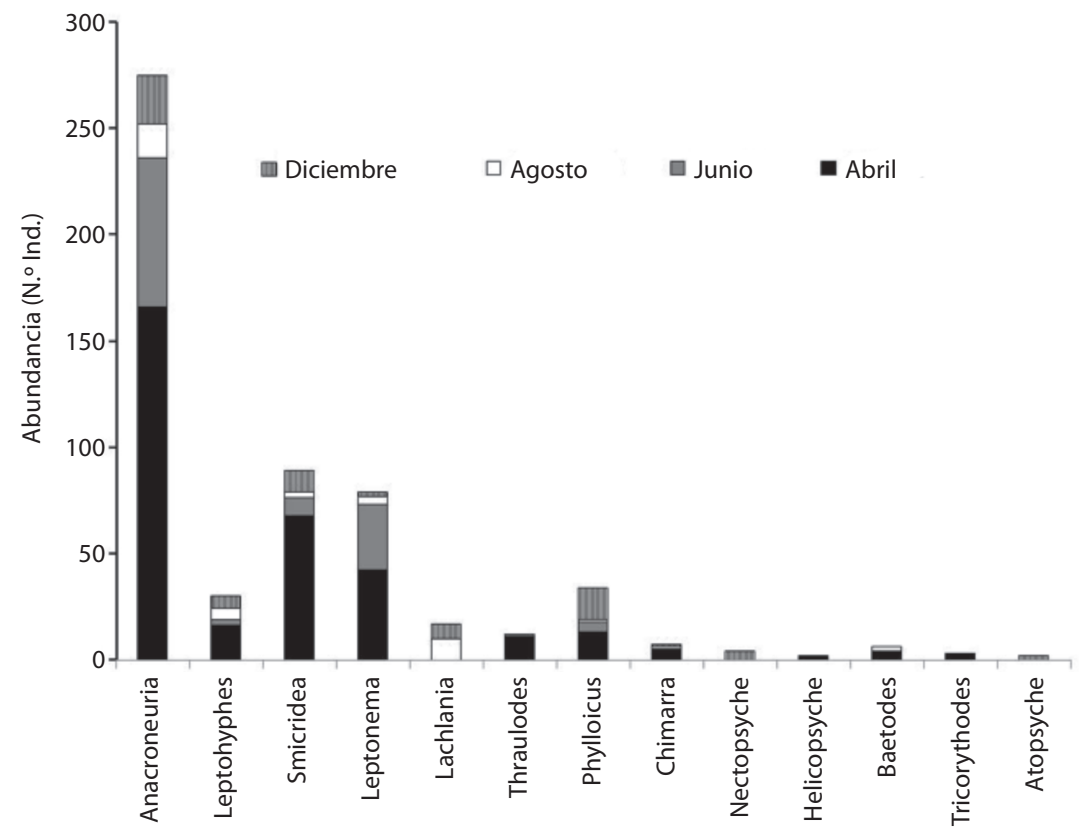

Fig. 2. Abundancia de los géneros de Ephemeroptera, Plecoptera y Trichoptera en abril, junio, agosto y diciembre de 2010 en la parte media del río Gaira, SNSM.

Fig. 2. Abundance of genera Ephemeroptera, Plecoptera and Trichoptera from middle reaches river Gaira, SNSM during April, June, August and December 2010.

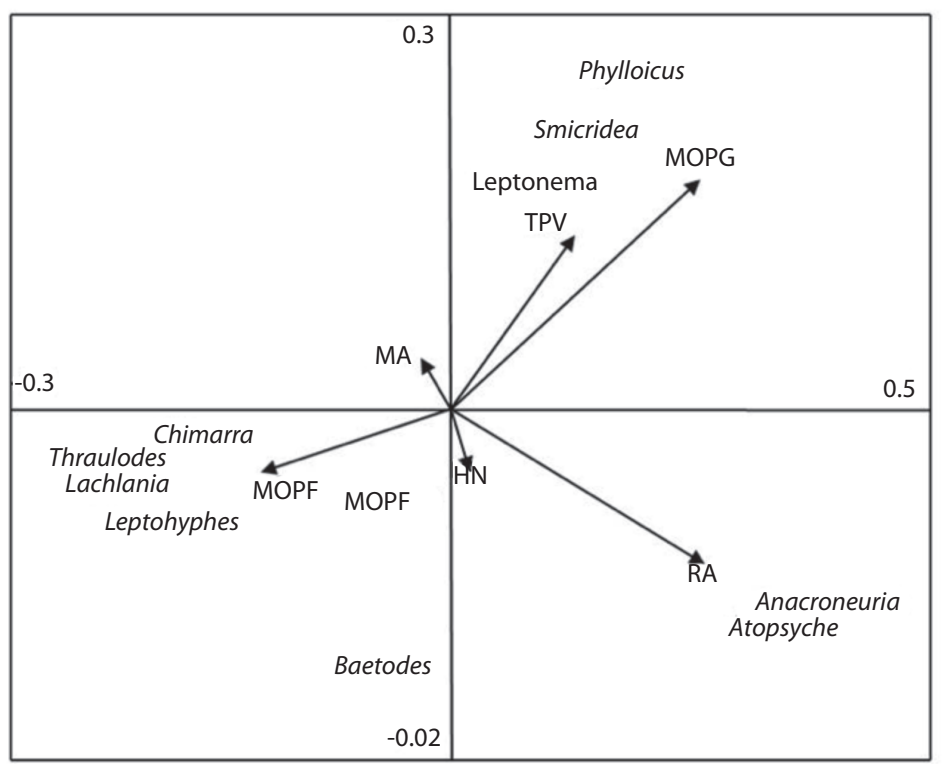

Fig. 3. Análisis de componentes principales (ACP) de diez géneros de Ephemeroptera, Plecoptera y Trichoptera presentes en la parte media del río Gaira, obtenido sobre la base de los ítems alimentarios identificados en organismos inmaduros evaluados.

Fig. 3. Principal component analysis (PCA) of ten genera of Ephemeroptera, Plecoptera and Trichoptera from middle reaches river Gaira, SNSM, obtained on the basis of food items identified. 
por Phylloicus, Smicridea y Leptonema, siendo MOPG y MOPF los ítems con mayor proporción promedio, por lo que son asignados, principalmente al gremio Detritívoro y al grupo funcional fragmentador para Phylloicus y recolector para los otros dos. El Grupo B está compuesto por Thraulodes, Lachlania, Chimarra y Leptohyphes, de los cuales su principal ítem alimentario fue MOPF en proporciones promedio mayores al $>80 \%$ asignándoseles al gremio Detritívoro, pero al grupo funcional Recolector. El Grupo C, en el que solo se encuentra Baetodes, con MOPF como principal ítem pero $<50 \%$ es también asignado a los Detritívoros, pero su grupo funcional sería principalmente como Recolector y Raspador de forma secundaria. Por ultimo, el Grupo D conformado por los géneros Anacroneuria y Atopsyche, géneros cuyo principal ítem alimentario ( $>50 \%$ ) fue RA, son categorizados como Depredadores (Fig. 3 y 4, Cuadro 2).
La prueba NPMANOVA detectó diferencias significativas $(p<0.05)$ entre las dietas de los géneros permitiendo inferir el comportamiento de los perfiles tróficos de los taxones estudiados y comprobar la diferencia que separa a cada grupo o la asignación secundaria. De esta forma, Anacroneuria y Atopsyche no presentaron diferencias significativas (NPMANOVA, $\mathrm{p}>0.05$ ), lo que sugiere un perfil dietario similar. Sin embargo, ambos géneros fueron significativamente diferentes de los otros grupos analizados $(\mathrm{p}<0.05)$. El género Baetodes presentó diferencias significativas con los demás taxones evaluados (NPMANOVA, $\mathrm{p}<0.05)$, esto evidencia la formación de un grupo conformado solo por éste género. Por otro lado, el análisis de comparación no detectó diferencias significativas entre los pares que conformarían Lachlania, Thraulodes y Leptohyphes (NPMANOVA, $\mathrm{p}>0.05$ ), por el contrario se detectó diferencia (NPMANOVA,

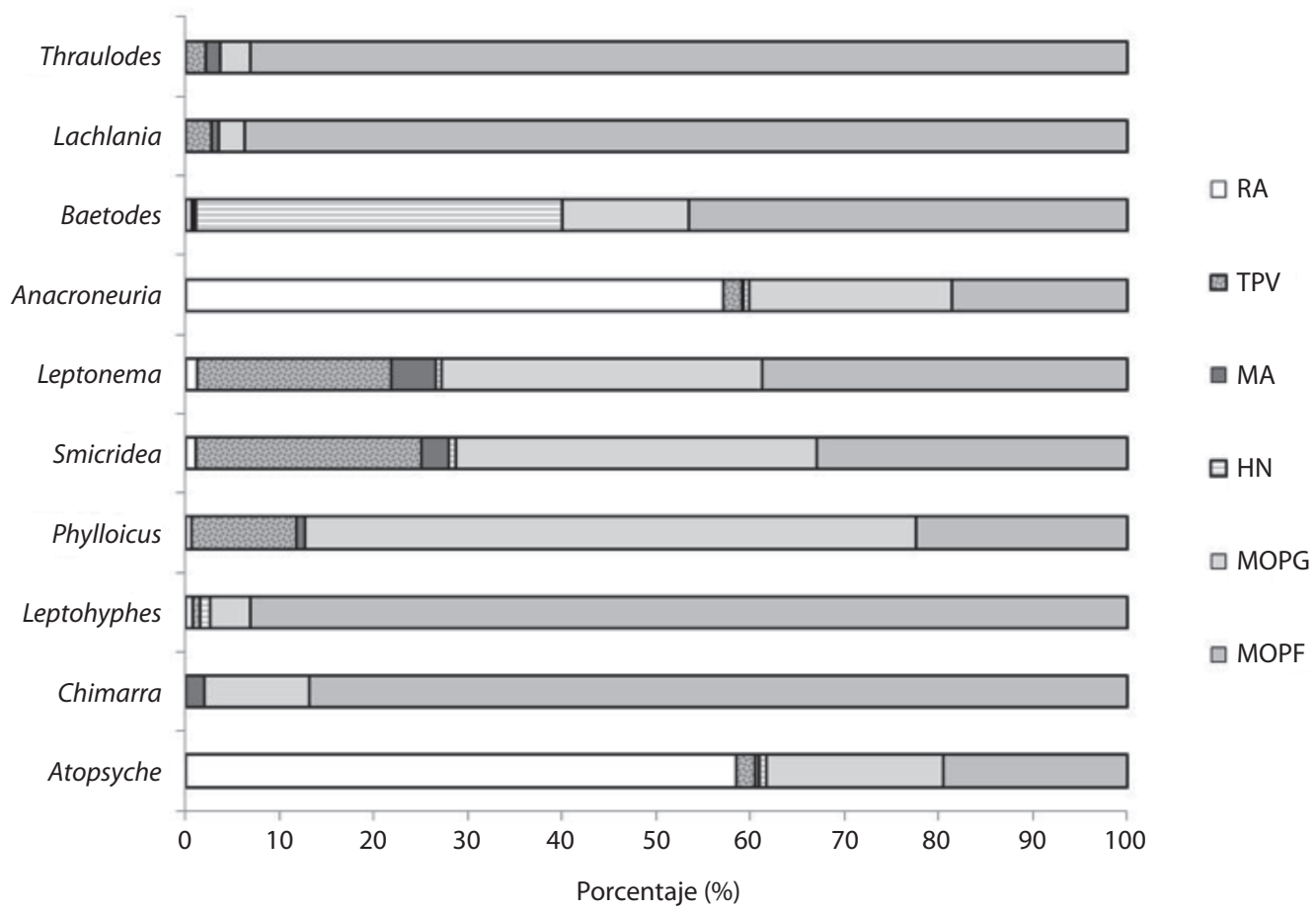

Fig. 4. Porcentaje promedio de cada tipo de alimento en organismos inmaduros de Ephemeroptera, Plecoptera y Trichoptera en la parte media del Río Gaira, SNSM.

Fig. 4. Average percent of each food resource of immature Ephemeroptera, Plecoptera and Trichoptera from middle reaches river Gaira, SNSM. 
CUADRO 2

Comparación de la clasificación de los hábitos alimentarios de los diez géneros analizado

TABLE 2

Classification comparison of the feeding habits of ten genera analyzed

\begin{tabular}{|c|c|c|c|c|}
\hline \multirow{2}{*}{ Orden } & \multirow{2}{*}{ Género } & \multicolumn{2}{|c|}{ (Presente trabajo) } & \multirow{2}{*}{ Otras referencias } \\
\hline & & GFA & GT & \\
\hline \multirow[t]{5}{*}{ Trichoptera } & Atopsyche & Depredador & Carnivoro & Depredador $^{1,2} ;$ Fragmentador-Generalista $^{3}$ \\
\hline & Chimarra & Recolector & Detritivoro & Recolector $^{1,2}$ \\
\hline & Phylloicus & Fragmentador-Recolector & Detritivoro & Fragmentador-Generalista $^{3} ;$ Recolector $^{1}$ \\
\hline & Smicridea & Fragmentador-Recolector & Detritivoro & Recolector $^{1,2} ;$ Fragmentador-Recolector ${ }^{3}$ \\
\hline & Leptonema & Fragmentador-Recolector & Detritivoro & Recolector $^{1,2}$; Fragmentador-Generalista ${ }^{3}$ \\
\hline Plecoptera & Anacroneuria & Depredador & Carnivoro & Depredador ${ }^{1,2,3}$ \\
\hline \multirow[t]{4}{*}{ Ephemeroptera } & Baetodes & Recolector-Raspador & Detritivoro & Raspador $^{1}$; Recolector ${ }^{2,3}$ \\
\hline & Lachlania & Recolector & Detritivoro & Recolector $^{1}$ \\
\hline & Thraulodes & Recolector & Detritivoro & Recolector $^{2}$ \\
\hline & Leptohyphes & Recolector & Detritivoro & Recolector $^{1,3}$ \\
\hline
\end{tabular}

1. Merritt \& Cummins 1996 (Norte América).

2. Tomanova et al. 2006 (Bolivia).

3. Chará et al. 2010 (Colombia, Risaralda).

GFA: grupo funcional alimentario y GT: gremio trófico.GFA, functional feeding group and GT: Trophic guild.

$\mathrm{p}<0.05$ ) entre Chimarra y todos los taxones restantes incluyendo a los Recolectores. Finalmente, entre las larvas de Smicridea y Leptonema no se detectó diferencia (NPMANOVA, $\mathrm{p}>0.05$ ), como si se observó entre Phylloicus y los otros taxones (NPMANOVA, $\mathrm{p}<0.05$ ).

Las diferencias entre Phylloicus con Smicridea y Leptonema apoyan la separación de estos dos grupos en cuanto a su función, ya que Phylloicus presentó proporciones promedio de MOPG y MOPF más altas que Smicridea y Leptonema, pero el menor valor promedio de TPV. Asimismo, entre el grupo de los Recolectores, la prueba de NPMANOVA detectó diferencias significativas (NPMANOVA, $\mathrm{p}<0.05)$ entre Chimarra con Lachlania, Thraulodes y Leptohyphes, siendo el primer género con la menor proporción promedio de MOPF (Cuadro 1).

\section{DISCUSIÓN}

Durante los muestreos se evidenció una baja abundancia de organismos (Fig. 2) con relación a otros estudios realizados en el mismo sitio (Tamarís-Turizo \& Sierra-Labastidas, 2009). Una posible explicación es debido a que se analizó un microhábitat (hojarasca) y los muestreos coincidieron con altas precipitaciones en el tramo de estudio, lo que evidencia que los cambios hidrológicos es un factor determinante en la composición y abundancias de los insectos acuáticos (Rincón, 1996; 2002; Tamarís-Turizo, Turizo-Correa \& Zúñiga, 2007; García-Alzate, Román-Valencia, González \& Barrero, 2010).

La MOPF fue el ítem alimentario más frecuente entre los organismos analizados, como ha sido demostrado en otros estudios (Palmer, O'Keeffe, Palmer, Dunney \& Radloff, 1993; Tomanova et al., 2006; Chará et al., 2010). Este recurso tiene gran importancia dentro de los sistemas acuáticos ya que es una de las principales fuentes energéticas para muchos organismos y es transportado rápidamente al río (Webster \& Benfield, 1986; Giller \& Malmqvist, 1998). En efecto, gran parte de la MOPF proviene de la MOPG (Allan \& Castillo, 2007), lo que implica una alta disponibilidad como recurso y explica su presencia como contenido estomacal en la 
gran mayoría de los organismos (Tomanova et al., 2006). Igualmente, es posible que en los depredadores esté presente debido a que estos pueden estar alimentados de otros organismos que ingieren MOPF como alimento principal, lo que explicaría la presencia de MOPF encontrado en Anacroneuria y Atopsyche.

Ninguno de los géneros presentó una dieta exclusiva por uno de los recursos categorizados, debido a que presentaron por lo menos tres ítems alimentarios. Si bien es cierto que los insectos acuáticos son oportunistas (Motta \& Uieda, 2004), se observó que Chimarra presentó los ítems MA, MOPG y MOPF, aunque es posible que las microalgas sean accidentales, debido a que se asientan sobre algún recurso que este género consuma (Tomanova et al., 2006; Allan \& Castillo, 2007). Lo cual se constituye en una evidencia de la plasticidad alimentaria en estos grupos de insectos acuáticos.

La categorización de Atopsyche y Anacroneuria como depredadores coincide con otros estudios (Merritt \& Cummins, 1996; Tomanova et al., 2006; Reynaga \& Rueda, 2010). Chará et al. (2010) clasificó al género Atopsyche como Fragmentador-Generalista, cuya diferencia en los hábitos alimentarios podría ser detectado a nivel de especie. Baetodes en EEUU fue registrado como Raspador exclusivo de microalgas (Merritt \& Cummins, 1996), lo cual contrasta con este trabajo, determinado como Recolector-Raspador, evidenciado por las altas proporciones de MOPF y hongos, este último recurso es considerado como material asociado al perifiton (Gulis \& Suberkropp, 2006). Estas diferencias en los hábitos pueden estar relacionadas con la disponibilidad de los recursos durante determinada época del año.

En este trabajo los cinco géneros de Trichoptera fueron representados en tres gremios tróficos, evidenciando el importante papel que cumplen dentro de las redes tróficas fluviales (Wiggins \& Mackay, 1978; Yule, 1996). Especial en procesos de descomposición de MOPG, generación de MOPF y en la depredación (Webster \& Benfield, 1986; Giller \& Malmqvist, 1998; Allan \& Castillo, 2007). Por otro lado, los géneros Lachlania, Thraulodes, Leptohyphes y Chimarra se consideraron Recolectores. Aunque éste último presentó diferencia significativa (NPMANOVA, $\mathrm{p}<0.05$ ) en comparación con los anteriores, lo que coincide con otros trabajos (Tomanova et al., 2006; Chará et al., 2010) lo que puede deberse a la afinidad por el recurso principal o al perfil dietario. Cheshire et al. (2005) sugieren que especies taxonómicamente relacionadas pueden tener dietas que difieren entre diferentes zonas, por tanto, un mismo taxón puede cambiar de gremio alimentario a lo largo de su distribución geográfica. Leptonema se registró como Detritívoro y Recolector aunque Chará et al. (2010) lo asignaron como Fragmentador-Generalista. Sin embargo, esto puede deberse a asignar grupos funcionales basados en dieta y no en modo de alimentación, como lo hace la categorización por Merritt \& Cummins (1996).

Reynaga (2009) asignó a Smicridea como Depredador-Recolector, y a Chimarra como Recolector-Filtrador con tallas diferentes en relación al presente trabajo, donde el primer grupo difiere de asignación y el segundo coincide. Smicridea es un filtrador, por lo que la presencia de presas en el contenido estomacal se puede deber a organismos en deriva. En este estudio encontramos que se alimenta más bien de detrito, y también se asigna como Recolector al alimentarse filtrando el agua. Del mismo modo, Atopsyche es reportado con tallas similares por Reynaga \& Rueda (2010) sin diferir la asignación al gremio trófico. Las diferencias en tallas pueden resultar en cambios en la dieta a medida que aumenta el tamaño del organismo (Cummins \& Klug, 1979; Céréghino, 2006).

En conclusión, los hábitos alimentarios en algunos EPT difieren entre ríos, posiblemente esta variabilidad de los hábitos puede estar relacionada con la disponibilidad de recursos alimentarios, microhábitats en el sistema, el estado de desarrollo del individuo, la composición de la comunidad y el régimen hidrológico (Merrit \& Cummins, 1996; Muñoz, Romaní, Rodríguez-Capitulo, Gonzales-Esteban \& García-Berthou, 2009; Chará et al., 2010). 


\section{AGRADECIMIENTOS}

A la Universidad del Magdalena, que a través de la Vicerrectoría de Investigaciones financió este proyecto en su totalidad, en la primera convocatoria para financiar proyectos en marco del programa de semilleros de investigación 2009-2011, a los integrantes del Grupo de Investigación en Limnología Neotropical y a los evaluadores anónimos por los aportes realizados al manuscrito.

\section{RESUMEN}

Los aspectos morfológicos y de comportamiento de insectos permiten agruparlos en gremios, y representar la dependencia de la comunidad lotica hacia recursos alimentarios particulares. Se evaluó los hábitos alimentarios de organismos inmaduros de Ephemeroptera, Plecoptera y Trichoptera (EPT) de la parte media del río Gaira (Sierra Nevada de Santa Marta, Colombia), por medio del análisis del contenido estomacal. Se identificaron 13 géneros de EPT, pero solo a diez de éstos se les revisó el contenido estomacal, para un total de 100 organismos analizados. Se describieron seis ítems alimentarios: restos animales (RA), tejido de plantas vasculares (TPV), Microalgas (MA), hongos $(\mathrm{HN})$, materia orgánica particulada gruesa (MOPG) y materia orgánica particulada fina (MOPF). Baetodes se registró como Recolector-Raspador, ya que su principal ítem alimentario fue MOPF (46.6\%) seguido de HN (38.4\%). Chimarra, Leptohyphes, Thraulodes y Lachlania, se categorizaron como Recolectores con proporciones promedio de $86.8 \%, 93.1 \%, 93.1 \%$ y $93.7 \%$ de MOPF, respectivamente. En los géneros Phylloicus, Leptonema y Smicridea se observó principalmente TPV y MOPG con $76.3 \%, 54,6 \%$ y $62.4 \%$ junto con proporciones de MOPF de $22.3 \%$, 38.8\% y $32.9 \%$, respectivamente. Categorizado como Detritívoro, Phylloicus es funcionalmente clasificado como Fragmentador y Leptonema y Smicridea como Recolectores. Los géneros Anacroneuria y Atopsyche fueron clasificados como Depredadores y fueron los únicos taxones en los que se observó RA en altas proporciones (57.9\% y $58.2 \%$ ). Nuestro estudio sugiere que los organismos examinados ingieren una amplia variabilidad de recursos.

Palabras claves: insectos acuáticos, contenido estomacal, ítem alimentario, grupos tróficos, río Gaira.

\section{REFERENCIAS}

Allan, J. D., \& Castillo, M. M. (2007). Detrital energy sources. In J. Allan \& M. M. Castillo (Eds.), Stream ecology structure and function of running waters (pp 135-161). Netherlands: Springer Dordrecht.
Anderson, M. J. (2001). A new method for non-parametric multivariate analysis of variance. Austral Ecology, 26, 32-46.

Céréghino, R. (2006). Ontogenetic diet shifts and their incidence on ecological processes: a case study using two morphologically similar stoneflies (Plecoptera). Acta Oecologica, 30, 33-38.

Chará-Serna, A. M., Chará, J. D., Zúñiga, M. C., Pedraza, G. X., \& Giraldo, L. P. (2010). Clasificación trófica de insectos acuáticos en ocho quebradas protegidas de la ecorregión cafetera colombiana. Universitas Scientiarum, 15(1), 27-36.

Cheshire, K., Boyero, L. U., \& Pearson, R. (2005). Food webs in tropical Australian streams: shredders are not scarce. Freshwater Biology, 50, 748-769.

Clarke, K. R., \& Gorley, R. N. (2005). PRIMER v5: User manual/ tutorial. Plymouth: Primer-E Ltd.

Cummins, K. M. (1973). Trophic relations of aquatic insects. Annual Review of Entomology, 18, 183-203.

Cummins, K. W., \& Klug, M. J. (1979). Feeding ecology of stream invertebrates. Annual Review of Ecology and Systematics, 10, 147-172.

Darrigan, G., Vilches, A., Legarralde, T., \& Damborenea, C. (2007). Guía para el estudio de macroinvertebrados: Métodos de colecta y técnicas de fijación. Argentina: ProBiota-FCN \& M-UNLP, Universidad de la Plata.

Domínguez, E., \& Fernández, R. (2009). Macroinvertebrados bentónicos sudamericanos: sistemática $y$ biología. Tucumán: Fundación Miguel Lillo,

Domínguez, E., Molineri, C., Pescador, M., Hubbard, M., \& Nieto, C. (2006). Ephemeroptera of South America. In J. Adis, J. R. Arias, S. Golovatch, K. M. Wantzen \& G. Rueda-Delgado (Eds.), Aquatic Biodiversity of Latin American-ABLA Vol 2 (pp. 646). Sofia-Moscow: Pensoft.

Fernández, H., \& Domínguez, E. (2001). Guía para la determinación de los artrópodos bentónicos sudamericanos. Tucumán: Fundación Miguel Lillo.

García-Alzate, C. A., Román-Valencia, C., González, M. I., \& Barrero, A. M. (2010). Composición y variación temporal de la comunidad de insectos acuáticos (Insecta) en la quebrada Sardineros, afluente Rio Verde, Alto Cauca, Colombia. Revista de Investigaciones de la Universidad de Quindio, 21, 21-28.

Giller, P. S., \& Malmqvist, B. (1998). The biology of Streams and Rivers. New York: Oxford University Press Inc.

Gulis, V., \& Suberkropp, K. (2006). Fungi: biomass, production and sporulation of aquatic hyphomycetes. En F.R. Hauer \& G.A. Lamberti (Eds.), Methods in stream ecology (pp. 311-325). New York: Academic Press.

Hammer, Ø., Harper, D. A. T., \& P. D. Ryan. (2001). PAST: Paleontological Statistics Software Package 
for Education and Data Analysis. Palaeontologia Electronica, 4(1), 9.

Legendre, P., \& Legendre, L. (1998). Numerical ecology. Amsterdam: Elsevier Publishers.

Merritt, R. W., \& Cummins, K. W. (1996). An introduction to the aquatic insects of North America. Iowa: Kendall-Hunt Publishing Company.

Morse, J. (2011). The Trichoptera world checklist. Zoosymposia, 5, 372-380.

Motta, R. L., \& Uieda, V. S. (2004). Diet and trophic groups of an aquatic insect community in a tropical stream. Brazilian Journal of Biology, 64(4), 809-817.

Muñoz, I., Romaní, A. M., Rodríguez-Capitulo, A., Gonzales-Esteban, J., \& García-Berthou, E. (2009). Relaciones tróficas en el ecosistema fluvial. In A. Elosegi \& S. Sabater (Eds.), Conceptos y técnicas en el ecosistema fluvial (pp. 347-366). España: fundación BBVA.

Muñoz-Quesada, F. (2004). El Orden Trichoptera (Insecta) en Colombia, II: inmaduros y adultos, consideraciones generales. In F. Fernández (Ed.) Insectos de Colombia, Vol. 3 (pp. 319-349). Bogotá: Universidad Nacional de Colombia.

Palmer, C., O'Keeffe, J., Palmer, A., Dunney, T., \& Radloff, S. (1993). Macroinvertebrate functional feeding groups in the middle and lower reaches of the Buffalo River, Eastern Cape, South Africa: Dietary variability. Freshwater Biology, 29, 441-453.

Pes, A. M. O., Hamada, N., \& Nessimian, J. L. (2005). Chaves de identificação de larvas para familias de Trichoptera (Insecta) de Amasônia Central, Brasil. Revista Brasileira de Entomología, 49(2), 181-204.

Posada, G. J., \& Roldán., G. (2003). Clave ilustrada y diversidad de larvas de Trichoptera en el Noroccidente de Colombia. Caldasia, 25(1), 169-192.

Reynaga, M. C., \& Rueda, M. P. (2010). Trophic analysis of two species of Atopsyche (Trichoptera: Hydrobiosidae). Limnologica, 40, 61-66.

Reynaga, M. C. (2009). Hábitos alimentarios de larvas de Trichoptera (Insecta) de una cuenca subtropical. Ecología Austral, 19, 207-214.

Rincón, M. E. (1996). Aspectos bioecológicos de los tricópteros de la quebrada Carrizal (Boyacá. Colombia). Revista Colombiana de Entomología, 22(1), 53-60.

Rincón, M. E. (2002). Comunidad de insectos acuáticos de la quebrada Mamarramos (Boyacá, Colombia). Revista Colombiana de Entomología, 28(1), 1001-108.

Sneath, P. H., \& Sokal, R. R. (1973). Numerical taxonomyThe principles and practice of numerical classification. San Francisco: Freeman and Company.

Sokal, R. R., \& Rohlf, F. J. (1995). Biometry: the principles and practice of statistics in biological research. $3 \mathrm{ed}$. New York: Freeman and Company
Stark, B. P., Froehlich, C., \& Zúñiga, M. C. (2009). South American Stonflies (Plecoptera), In J. Adis, J. R. Arias, S. Golovatch, K. M. Wantzen \& G. RuedaDelgado (Eds.), Aquatic Biodiversity of Latin American- $A B L A$. Vol 5 (pp. 154) Sofia-Moscow: Pensoft.

Stewart, K. W., \& Stark, B. P. (2002). Perlidae-The Stonesfly. In K.W. Stewart \& B. P. Stark (Eds.), Nymphs of North American Stonefly Genera (Plecoptera). 2ed (pp. 320-323). Ohio: The Caddis Press.

Tamarís-Turizo, C., Turizo-Correa, R., \& Zúñiga, M. C. (2007). Distribución espacio-temporal y hábitos alimentarios de ninfas de Anacroneuria (Insecta: Plecoptera: Perlidae) en el río Gaira (SNSM, Colombia). Caldasia, 29(2), 375-385.

Tamarís-Turizo, C., \& Sierra-Labastidas, T. (2009). Una inspección al papel de la producción de los Plecópteros en ecosistemas lóticos. Revista de Ciencias, 12, 109-120.

Tomanova, S., Goitia, E., \& Helesic, J. (2006). Trophic levels and functional feeding groups of macroinvertebrates in neotropical streams. Hydrobiologia, 556, 251-264.

Townsend, C. R., \& Hildrew, A. G. (1994). Species traits in relation to a habitat templet for river systems. Freshwater Biology, 31, 265-275.

Vannote, R. L., Minshall, G. W., Cummings, K. W., Sedell, J. R., \& Cushing, C. E. (1980). The river continuum concept. Canadian Journal of Fisheries and Aquatic Sciences, 37, 130-137.

Wantzen, K. M., \& Rueda, G. D. (2009). Técnicas de muestreo de macroinvertebrados bentónicos. In E. Domínguez \& H. R. Fernández (Eds.), Macroinvertebrados bentónicos sudamericanos: sistemática y biología. (pp. 17-45). Tucumán: Fundación Miguel Lillo.

Webster, J. R., \& Benfield, E. F. (1986). Vascular plant breakdown in freshwater ecosystems. Annual Review of Ecological and Systematic, 17, 567-594.

Wiggins, G. B., \& Mackay, R. J. (1978). Some relationships between systematics and trophic ecology in neartic aquatic insects, with special reference to Trichoptera. Ecology, 59, 1211-1220.

Wiggins, G. B. (1996). Larvae of the North American caddisfly genera. 2ed. Toronto: University of Toronto Press.

Wiggins, G. B. (2004). Caddisflies. The underwater architecs. Toronto: University of Toronto Press.

Yule, C. M. (1996). Trophic relationship and food webs of the benthic invertebrate fauna of two seasonal tropical streams on Bougainville Island, Papua Nueva Guinea. Journal of Tropical Ecology, 12, 517-534.

Zúñiga, M. C. 2010. Diversidad, distribución y ecología del orden Plecoptera (Insecta) en Colombia, con énfasis en Anacroneuria (Perlidae). Momentos de Ciencia, $7(2), 101-112$. 